\title{
STRATEGY ON SIMULATION OF ARBITRARY-SHAPED CEMENT GRAINS IN CONCRETE
}

\author{
Huan He, Zhanqi Guo, Piet Stroeven, Martijn Stroeven and Lambertus Johannes \\ SLUYS
}

Faculty of Civil Engineering and Geosciences, Delft University of Technology, Stevinweg 1, 2628CN Delft, Netherlands

e-mail: H.He@live.com

(Accepted March 2, 2010)

\begin{abstract}
The shape of cement particles plays an important role in the hydration process due to surface dissolution and hardening process. Nevertheless, a spherical particle shape is normally assumed in conventional simulation systems, because of the inherent simplification in algorithm formulations and corresponding reduced computer time, however, at the cost of possibly biased simulation results. Recently, some reference cement was analyzed by X-ray micro-tomography. The results provide a real experimental database of this cement that yields valuable parameters for simulation of cement hydration. A shape analysis study was conducted with some simpler shape solutions, whereby the X-ray micro-tomography results served as a reference. Based on this analysis, a simulation strategy is proposed as a preferred approach to cement particle simulation. The generation of the densely packed microstructure of fresh cement paste in this study was conducted by using an advanced discrete element method (DEM).
\end{abstract}

Keywords: cement grains, discrete element method, shape analysis, X-ray micro-tomography.

\section{INTRODUCTION}

Concrete is made of hard inclusions surrounded by a hydraulic, cementitious matrix. Bonding between aggregate and hardened matrix provides concrete with a capacity to resist tensile stresses. Properties of the cement in addition to mixture design and curing conditions control the mechanical behavior of concrete. The shape of cement particles plays also an important role in the hydration process and structure formation, including that of pore space. Cement hydration in a DEM approach renders possible investigating microstructure development and properties of concrete. A digital image-based model (Bentz, 1997) as well as continuum models (van Breugel, 1997; Navi and Pignat 1999; Stroeven, 1999) have been developed for this purpose. Apart from the digital-image-based model, particle shape is generally assumed spherical in such systems, because of inherent simplification in algorithm formulation; however, at the cost of possibly biased simulation results.

Computer X-ray micro-tomography offers a potential solution for shape assessment of cements, as shown by Garboczi and Bullard (2004) and by Bullard and Garboczi (2006). The microstructures of hydrated cements based on actual grain shape and on spherical shape were found significantly different in this study. The results provide an experimental database of this cement that yields some valuable parameters for DEM simulation of cement hydration.

Using grains in numerical simulation similar to the real ones would be too expensive. So, it is crucial finding simpler shapes that are sufficiently representative. A shape analysis study was therefore conducted with some simpler shapes. Based on this analysis, a simulation strategy is proposed for cement. Implementation of this simulation strategy in the concurrentalgorithm-based particle packing simulation system HADES as well as some packing simulations are also discussed in this paper.

\section{EXPERIMENTAL REFERENCE BY X- RAY MICRO-TOMOGRAPHY}

Garboczi and Bullard (2004) analyzed a standard reference cement, CCRL-133, with Blaine fineness of about $350 \mathrm{~m}^{2} / \mathrm{kg}$. Synchrotron based X-ray imaging resolution was $0.95 \mu \mathrm{m}$ per voxel. Clear images of cement particles were obtained after 3 hours of hydration. It was assumed that the obtained shape of cement particles by X-ray micro-tomography was close to that of the initial particles (Garboczi and 
Bullard, 2004). Cement particles finer than $1 \mu \mathrm{m}$ were not included in this study due to limited resolution. A spherical harmonic (SH) (Garboczi, 2002) analysis was applied for shape reconstruction and surface estimation. The results provide an experimental database of cement, including some important parameters for cement hydration. Fig. 1a offers views on two typical cement particles. Fig. 1b shows S/V-data (correlation of surface area (S) and volume (V)) of reference cement particles and of spheres, differences being quite obvious. The regression line for the real cement particles is given by, with a correlation coefficient of 0.98 . for spheres. The surface area of cement particles is also sizedependent: larger grains have a lower coefficient of, i.e., tending to a sphere.
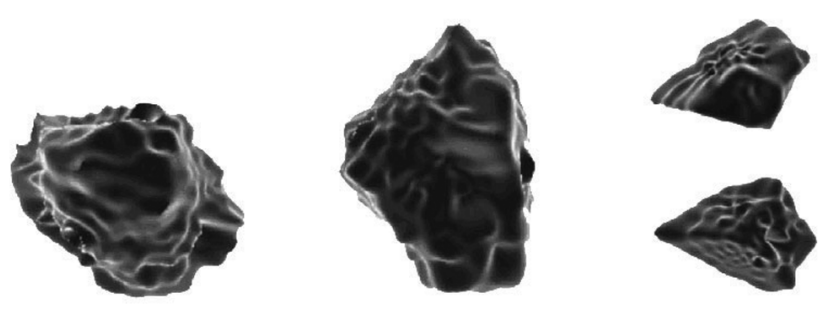

a)

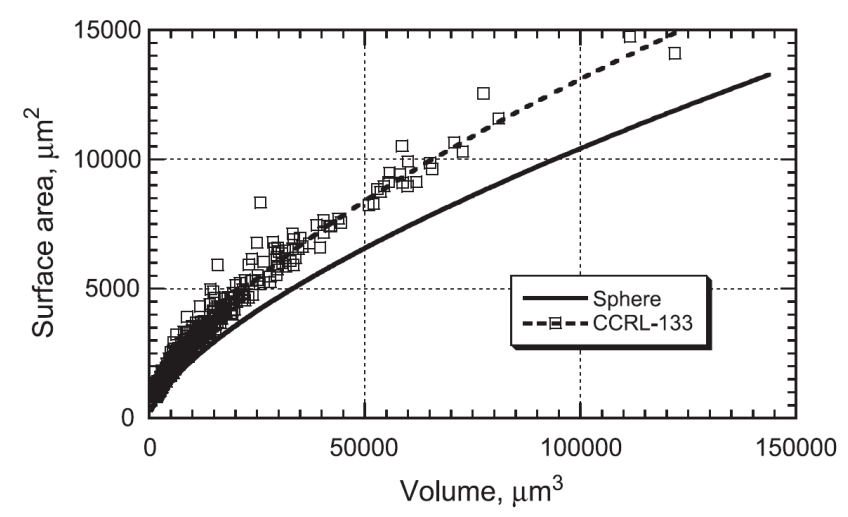

b)

Fig. 1. a) Two views of a large and a small cement particle; b) Surface area versus volume for all 1200 CCRL-133 cement particles analyzed (after Garboczi and Bullard, 2004).

\section{SHAPE ANALYSIS}

The spherical grain shape is commonly adopted in conventional cement hydration simulation systems (van Breugel, 1997; Navi and Pignat, 1999; Stroeven, 1999). However, the aforementioned experiments by $\mathrm{X}$-ray micro-tomography have shown that this is a somewhat biased approach. More realistic but still cost-effective particle shapes should therefore be implemented in DEM systems.

\section{Analysis of some polyhedra}

The polyhedron is a potentially promising choice for particle shape. Nine polyhedra with facet numbers from 4 to 8 are selected in this preliminary shape analysis (see, Fig. 2). As the S/V-relationship was the most important information obtained from the experimental reference, values of these shape indices are listed in Table 1 and plotted in Fig. 3. Sphericity is defined as surface area ratio of sphere and particle (with similar volume). The $\mathrm{S} / \mathrm{V}$-relationship can generally be expressed by (a is coefficient). Within effective experimental volume range of 10,000 150,000 $\mu \mathrm{m} 3$, coefficient was found by regression, with a coefficient of variation lower than 5\%. Proposed shapes should therefore approximate this value. In this case, sphericity can be calculated by

$$
\text { Sphericity }=\frac{S_{\text {eq.sphere }}}{S_{\text {particle }}}=\frac{4 \pi\left(\frac{3 V}{4 \pi}\right)^{2 / 3}}{a V^{2 / 3}} \approx 0.83 .
$$

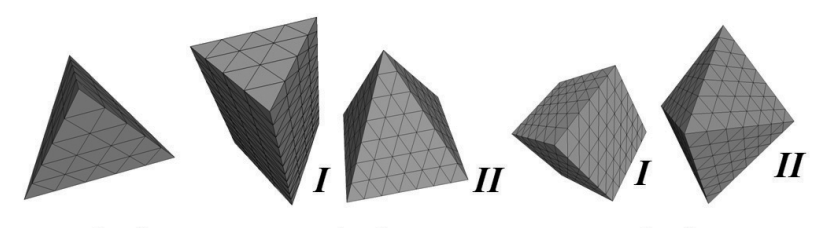

Tetrahedron Pentahedron

Hexahedron

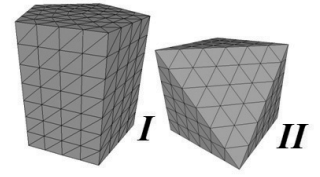

Heptahedron

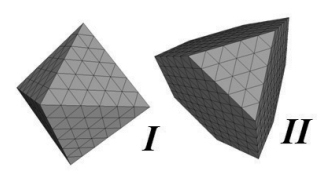

Octahedron
Fig. 2. Nine regular polyhedra with facet numbers $4 \sim 8$.

From Table 1 and Fig. 3 it is concluded that heptahedron I and octahedron I offer the most promising solutions. The octahedron is selected for the simulation study because of allowing an easier transformation into irregular shapes by parameter variation. Three axes can be employed for this purpose, as in He et al. (2008). Since the S/V-curve of octahedron I is close to that of the reference cement, some limited random variation in axes will be necessary. 
Table 1. Comparison of shape indices of regular polyhedra and spheres (S: surface area, V: volume).

\begin{tabular}{lccc}
\hline Shapes & Facet number & Sphericity & $S / V^{2 / 3}$ \\
\hline Tetrahedron & 4 & 0.67 & 7.21 \\
Pentahedron I & 5 & 0.70 & 6.95 \\
Pentahedron II & 5 & 0.72 & 6.71 \\
Hexahedron & 6 & 0.76 & 6.39 \\
Cube & 6 & 0.81 & 6.00 \\
Heptahedron I & 7 & $\mathbf{0 . 8 3}$ & $\mathbf{5 . 8 3}$ \\
Heptahedron II & 7 & 0.80 & 6.06 \\
Octahedron I & 8 & $\mathbf{0 . 8 5}$ & $\mathbf{5 . 7 2}$ \\
Octahedron II & 8 & 0.78 & 6.24 \\
Sphere & $\infty$ & 1.00 & 4.84 \\
\hline
\end{tabular}

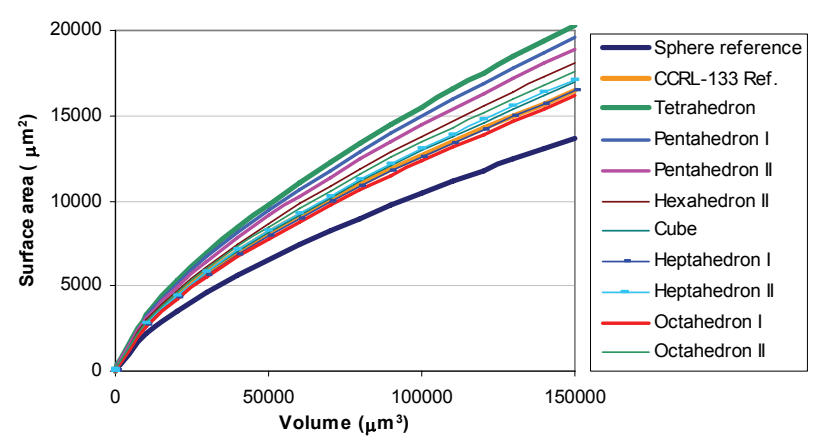

Fig. 3. Surface area versus volume curves of reference cement, sphere and some regular polyhedra.

\section{Analysis of ellipsoids}

Ellipsoids offer another promising possibility. Moreover, this shape can be easily derived from a sphere. Three principal axes (a, b, c represent the longest, medium and shortest axis, respectively, as shown in Fig. 4) determine the shape of an ellipsoid. By parameter variation, different ellipsoids with different $\mathrm{S} / \mathrm{V}$-relationships can be derived. $\mathrm{V}$ and $\mathrm{S}$ can be obtained by (Weisstein, 2010):

$$
V=\frac{4}{3} \pi a b c .
$$

$$
\begin{aligned}
& S=2 b \sqrt{2} \int_{0}^{\pi} \sqrt{a^{2}+c^{2}+\left(a^{2}-c^{2}\right) \cos (2 \phi)} \sin \phi \\
& \times E\left(\frac{c}{b} \sqrt{\frac{2\left(b^{2}-a^{2}\right)}{a^{2}+c^{2}+\left(a^{2}-c^{2}\right) \cos (2 \phi)}} \sin \phi\right) d \phi
\end{aligned}
$$

in which is a coefficient of the first fundamental form; is a polar angle.

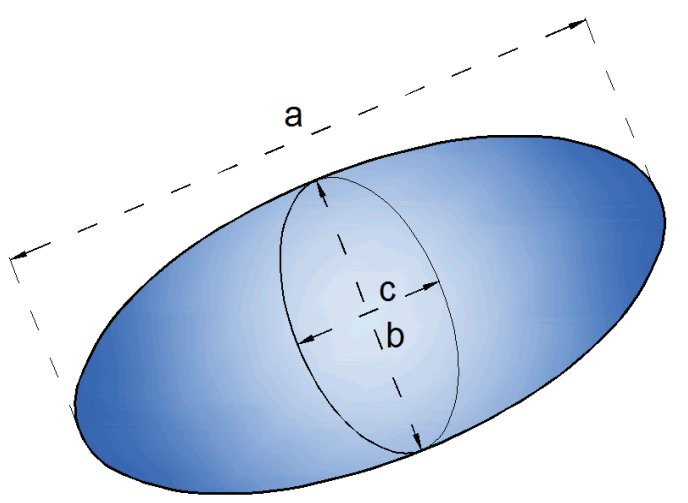

Fig. 4. An ellipsoid with three axes: $a, b, c$.

Flatness and elongation are defined as and, respectively. So, the distribution contours of sphericity with different elongation and flatness values can be constructed (see, Fig. 5).

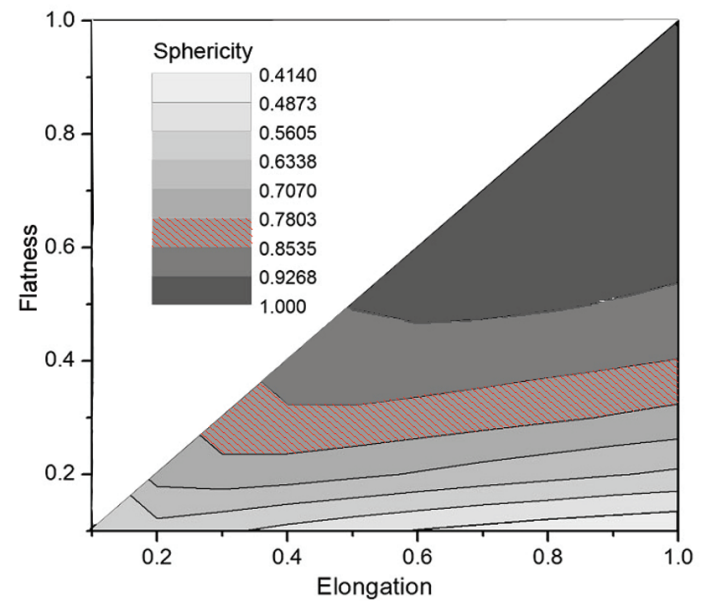

a)

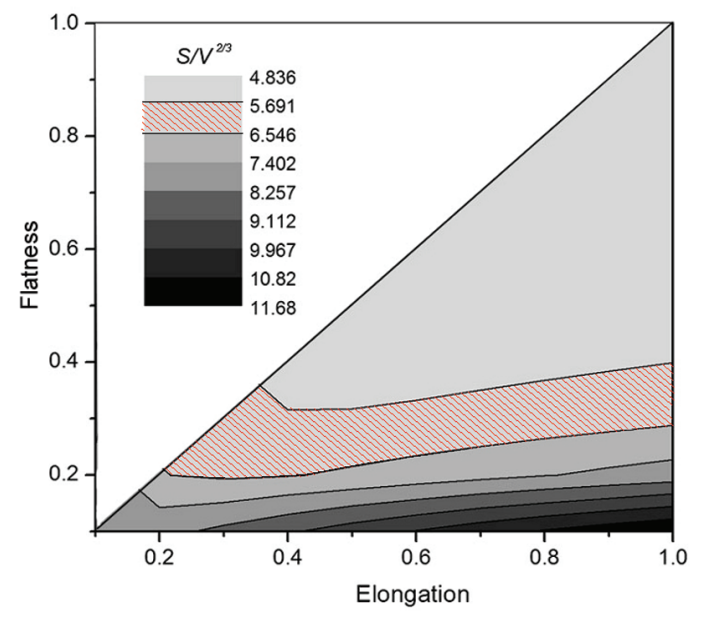

b)

Fig. 5. Distribution contours of a) sphericity and b) with different elongation and flatness of an ellipsoid. 
Because $\mathrm{c}<\mathrm{b}<\mathrm{a}$, elongation is always exceeding flatness. Fig. 5a reveals that if elongation and flatness are both larger than 0.5 , sphericity of an ellipsoid will be close to 1. -contours with elongation and flatness are plotted in Fig. 5b. The hatched region can be selected as optimum solution.

\section{IMPLEMENTATION OF THE SELECTED SHAPE IN DEM}

Modern high performance concretes are produced at low water to cement ratio: volume content of the cement in the paste may be as high as $60 \%$. The traditional DEM simulation system in concrete technology is using random generators to disperse cement (and mineral admixture) particles on microlevel in the watery environment during the fresh state. These systems are referred to as random sequential (particle) addition (RSA) systems. As stipulated elsewhere, they generate particle packing distinct from actual ones (Stroeven et al., 2008). Additionally, RSA systems cannot produce high densities. Concurrent algorithm-based simulation (CAS) systems were developed starting from RSA procedures, avoiding such limitations (Stroeven, 1999; Williams and Philipse, 2003; Stroeven et al., 2009). An advanced CAS system like HADES has been developed with capabilities of packing arbitrary shaped particles (He et al., 2008). The preferred shapes defined in this study can also be incorporated in HADES. After assessment of grain shape, mass properties are assigned to each particle, e.g., mass, centre of mass, moment of inertia. Some packing simulation examples are presented.

Fig. 6a illustrates S-V information of a group of simulated cement grains using flat ellipsoids. Data fit experimental results (Garboczi and Bullard, 2004) quite well.

A visual model of correspondingly loose packed ellipsoids obtained by HADES is plotted in Fig. 6 b.

Fig. 7 presents S/V-distributions of two examples of randomly generated octahedron grains with an experimental reference curve. Fig. 7a reveals the case of 1000 particles with longest axis in $10 \sim 50 \mu \mathrm{m}$ size range. The $\mathrm{S} / \mathrm{V}$-relationship complies well with experiments.

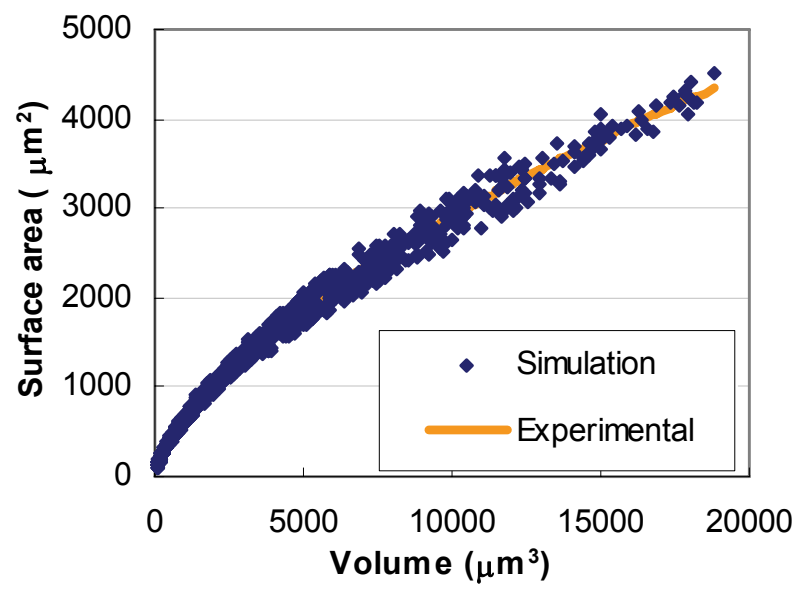

a)

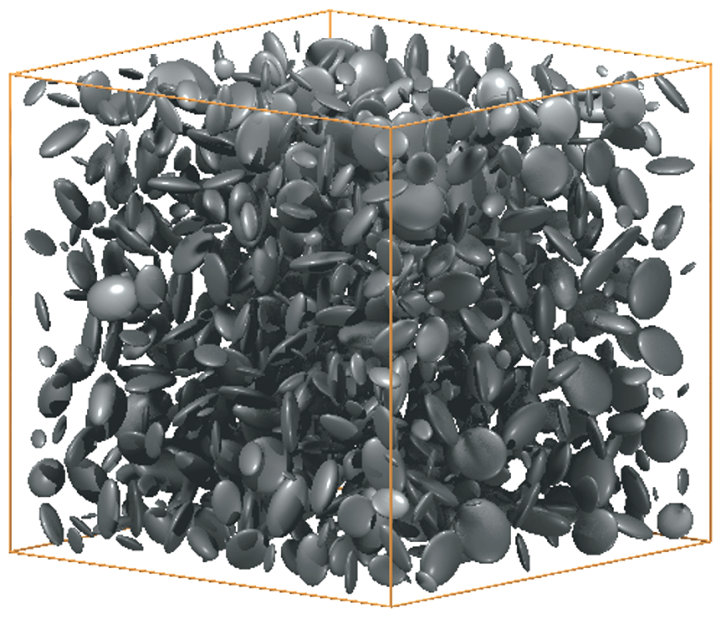

b)

Fig. 6. a) S-V-relationships of a group of cement grains by flat ellipsoids with experimental regression results; b) correspondingly packed visual model (1000 particles with longest axis in 10 50 $\mu \mathrm{m}$ range). 


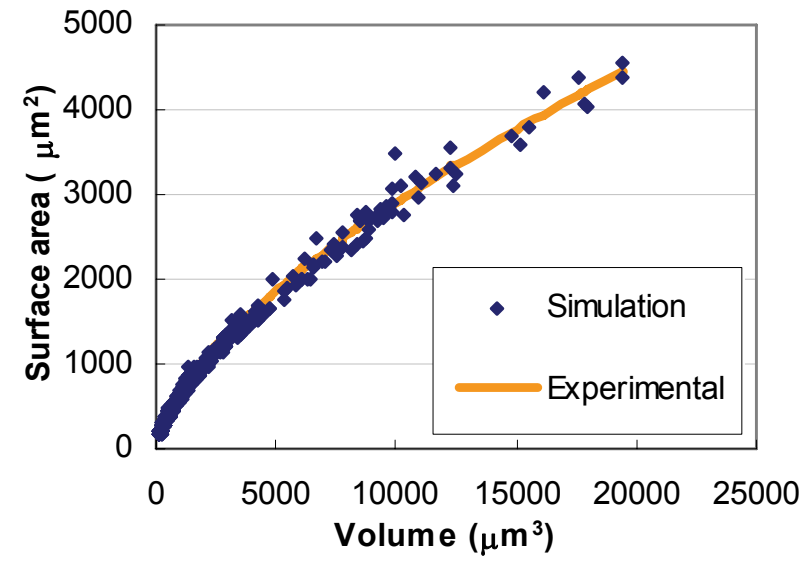

a)

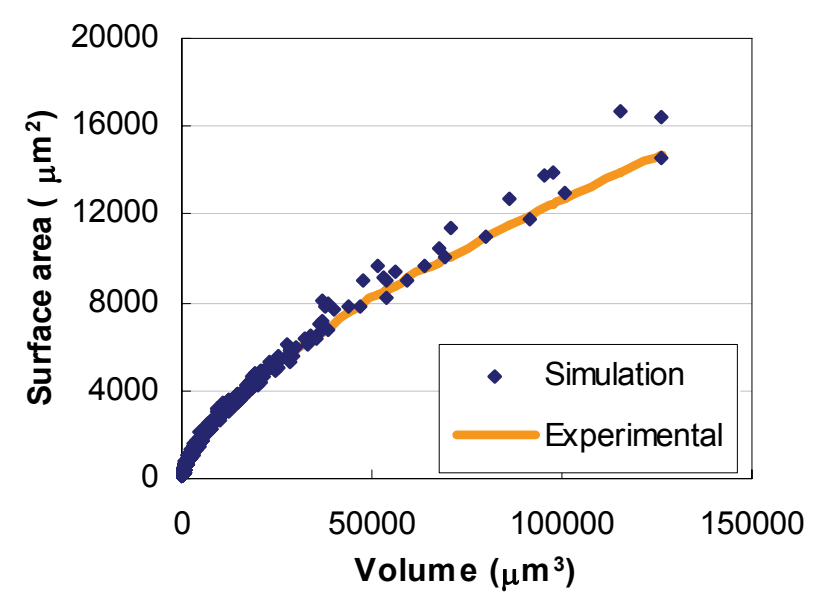

b)

Fig. 7. Computer simulation of octahedra packing and experimental regression results; a) 1000 particles with longest axis in 10 50 $\mu \mathrm{m}$ and b) 2000 particles with longest axis in 10 100 $\mu \mathrm{m}$ range.

But in the case of the larger particles in Fig. $7 \mathrm{~b}$ (maximum longest axis of particles is around 100 $\mu \mathrm{m})$, the simulated particles have a slightly higher surface area as in the experimental reference case. It indicates that larger particles tend to a spherical shape. One possible reason for this phenomenon is that those larger particles are to a smaller degree affected by grinding and thus less angular as a result.

Fig. 8a shows an example of a packed structure of arbitrary octahedrons and a section image in Fig. 8b. Packed structure of simulated cement particles with non-spherical shape can be used as fresh cement paste structure.

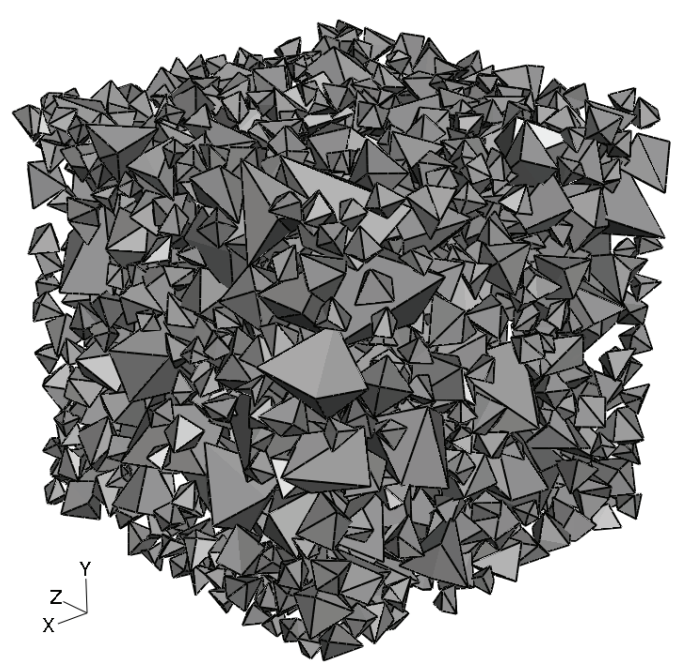

(a)

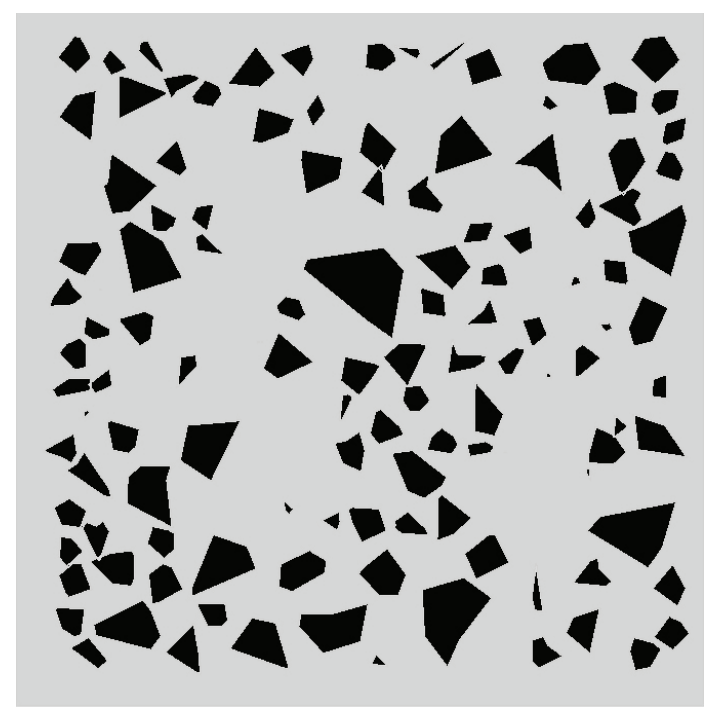

(b)

Fig. 8. a) Visual model of compacted grains and b) section of the simulated structure (1000 octahedron grains in 10 50 $\mu \mathrm{m}$ range).

To select the more reasonable shapes for simulation of cement particle grains, a review will focus on the production process of cement. Cement is made by raw materials such as limestone, clay, etc, after a process of calcination. Raw cement clinkers are hard, large and roughly rounded particles in scale of centimetres. Raw cement clinkers are ground in a miller with a small amount of gypsum into small cement grains. As demonstrated by Fig. 1a, final cement grains tend to angular shapes in scale of micrometers. Polyhedra are therefore most appropriate for simulation of cement. 
Morphological comparisons can additionally be made between sections of simulated particle structures and of real ones. As an example, Fig. 9 shows a 2D section of a real cement structure obtained by X-ray micro-tomography. Cement particles, displayed in light grey, obviously reveal the angular shape that is similarly revealed by the section of the polyhedral cement particles in Fig. 8b. This adds to the aforementioned quantitative matching of $\mathrm{S}-\mathrm{V}$ features of real and simulated cement structures, as shown by Fig. 7a. Therefore, it can be concluded that the proposed simulation method provides a significantly improved option for modelling of cement particles as compared to the conventional sphere system. This will also have impact on the hydration process, of course.

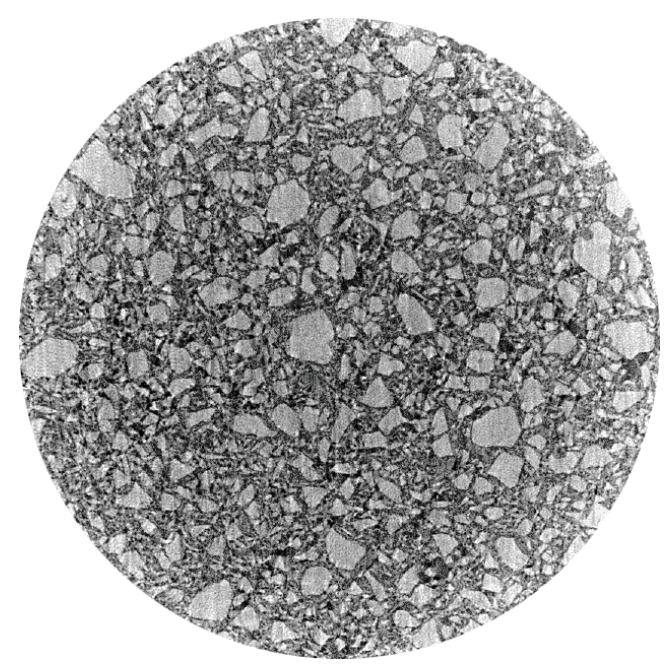

Fig. 9. 2D section of a real fresh cement structure (Cement-133) obtained by X-ray micro-tomography; $w / c=0.35$. Source: http://visiblecement.nist.gov/ cement.html.

\section{CONCLUSIONS}

For hydration simulation, a study was conducted pursuing the assessment of physically more realistic shapes as the commonly employed spherical one. This study encompassed polyhedra and ellipsoids. Experiments by X-ray micro-tomography on real cement were used as reference. It was shown that a limited variation of octahedrons or flat ellipsoids can provide optimized solutions, offering more realistic $\mathrm{S} / \mathrm{V}$-relationships as in the case of spheres, which is crucial for cement hydration (Bullard and Garboczi,
2006). Of the two particle shapes, the polyhedral one seems closer to the actual morphology of the cement particles. This simulation strategy was incorporated in the concurrent-algorithm based DEM system HADES, so that more accurate simulation of cement hydration can be realized.

\section{ACKNOWLEDGEMENTS}

The authors acknowledge Dr. E.J. Garboczi for his permission to reprint Fig. 1 from his publications.

\section{REFERENCE}

Bentz DP (1997). Three-dimensional computer simulation of portland cement hydration and microstructure development. J Amer Ceramic Soc 80:3-21.

Breugel K van (1997). Simulation of hydration and formation of structure in hardening cement- based materials. Delft: Delft University Press.

Bullard JW, Garboczi EJ (2006). A model investigation of the influence of particle shape on Portland cement hydration. Cem Concr Res 36:1007-15.

Garboczi EJ (2002). Three-dimensional mathematical analysis of particle shape using X-ray tomography and spherical harmonics: Application to aggregates used in concrete. Cem Concr Res 32:1621-38.

Garboczi EJ, Bullard JW (2004). Shape analysis of a reference cement. Cem Concr Res 34:1933-7.

He H, Guo Z, Stroeven P, Stroeven M (2008). Discrete element approach to packing of arbitrary shaped particles in concrete. Inżynieria Materiałowa 29:403-7.

Navi P, Pignat C (1999). Three-dimensional characterization of the pore structure of a simulated cement paste. Cem Concr Res 29:507-14.

Stroeven M (1999). Discrete numerical modeling of composite materials; application to cementitious materials. PhD Thesis, Delft University of Technology, Delft: Delft University Press.

Stroeven P, He H, Guo Z, Stroeven M (2008). Patchy nature of cement and concrete revealed by physical discrete element simulation approach. Inżynieria Materiałowa 29:408-12.

Stroeven P, Hu J, Stroeven M (2009). On the usefulness of discrete element computer modeling of particle packing for material characterization in concrete technology. Comp \& Concr 6:133-53.

Weisstein EW. Ellipsoid. http://mathworld.wolfram.com/ ellipsoid. html. Accessed May 24, 2010.

Williams SR, Philipse AP (2003). Random packings of spheres and spherocylinders simulated by mechanical contraction. Phys Rev E 67:1-9. 